Pacific Journal of Mathematics

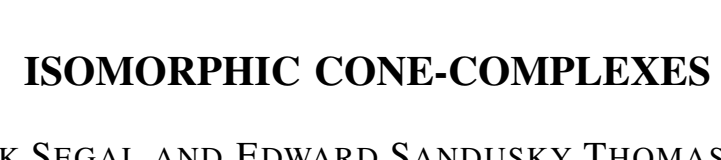




\title{
ISOMORPHIC CONE-COMPLEXES
}

\author{
JaCk Segal and E. S. Thomas, JR.
}

In this paper we show that the 1-section of a finite simplicial complex $M$ is characterized by the topological type of the 1-section of the cone over $M$. This enables us to prove that a finite simplicial complex is characterized by the topological type of the 1-section of the first derived complex of its cone.

R. L. Finney [1] proved that two locally-finite simplicial complexes are isomorphic if their first derived complexes are isomorphic. J. Segal [3] making use of this showed that two locally-finite simplicial complexes are isomorphic if the 1-sections of their first derived complexes are isomorphic. He then showed in [4] that a restricted class of finite complexes are characterized by the topological type of the 1section of their first derived complexes. In contrast to [4] the results of this paper apply without restricting the class of finite complexes.

Throughout, $s_{p}$ will denote a (rectilinear) $p$-simplex; $M$ will denote a finite geometric simplicial complex with $r$-section $M^{r}$ and first derived complex $M^{\prime}$. The cone at $m$ over $M, m \notin M$, is denoted by $m M$. For more details see $[2,1.2]$. We only consider complexes with at least two vertices.

Lemma. (a) If $m M$ and $n N$ are isomorphic then so are $M$ and $N$. (b) If $(m M)^{1}$ and $(n N)^{1}$ are isomorphic then so are $M^{1}$ and $N^{1}$.

Proof. (a) Let $\varphi$ be an isomorphism of $m M$ onto $n N$. If $\varphi(m)=n$ we are done so we assume $\varphi(m) \neq n$, hence we also have $\varphi^{-1}(n) \neq m$.

Given a complex $K$, with vertex $v$, the subcomplex consisting of those simplexes not having $v$ as a vertex is denoted $K\langle v\rangle$.

We now define subcomplexes of $M$ and $N$ as follows:

$$
\begin{array}{ll}
M_{1}=(m M)\left\langle\varphi^{-1}(n)\right\rangle \quad, M_{2}=M\left\langle\varphi^{-1}(n)\right\rangle \\
N_{1}=(n N)\langle\varphi(m)\rangle \quad, N_{2}=N\langle\varphi(m)\rangle .
\end{array}
$$

The following relationships are easily verified:

(1) $M_{1}=m M_{2}, N_{1}=n N_{2}$;

(2) $\varphi \mid M_{2}$ is an isomorphism of $M_{2}$ onto $N_{2}$;

(3) $\varphi \mid M$ is an isomorphism of $M$ onto $N_{1}$, and $\varphi^{-1 \prime} N$ is an isomorphism of $N$ onto $M_{1}$.

Using $\approx$ to denote isomorphism we then have: 


$$
M \approx N_{1}=n N_{2} \approx m M_{2}=M_{1} \approx N .
$$

Here the first and last isomorphisms follow from (3), the equalities from (1) and the middle isomorphism follows from (2) and the fact that taking cones preserves isomorphism.

A proof of part (b) is obtained by taking 1-sections at appropriate places in the above argument.

THEOREM 1. If $\left|(m M)^{1}\right|$ and $\left|(n N)^{1}\right|$ are homeomorphic then $M^{1}$ and $N^{1}$ are isomorphic.

Proof. For nontriviality we assume each of $M$ and $N$ has at least 3 vertices. Let $T_{M}$ denote the set of vertices of $m M$ whose order in $\left|(m M)^{1}\right|$ is not 2 and let $T_{N}$ be the corresponding set in $n N$; then $m$ is in $T_{M}$ and $n$ is in $T_{N}$.

Now let $h$ be a homeomorphism of $\left|(m M)^{1}\right|$ onto $\left|(n N)^{1}\right|$. We shall modify $h$ where necessary to get a new homeomorphism $\widetilde{h}$ which maps the vertices of $(m M)^{1}$ onto those of $(n N)^{1}$.

Clearly any homeomorphism of $\left|(m M)^{1}\right|$ onto $\left|(n N)^{1}\right|$ takes $T_{M}$ onto $T_{N}$. Let $v_{1}, \cdots, v_{r}$ be the vertices of $(m M)^{1}$ having order 2 ; we show how to construct homeomorphisms $h_{1}, \cdots, h_{r}$ such that

$$
h_{i}:\left|(m M)^{1}\right| \rightarrow\left|(n N)^{1}\right|
$$

and for $i \leqq j, h_{j}\left(v_{i}\right)$ is a vertex of $(n N)^{1}$. Starting with $h$ we shall construct $h_{1}$; the construction of $h_{i}$ from $h_{i-1}$ follows the same pattern and will be omitted.

For simplicity we write $v$ rather than $v_{1}$. If $h(v)$ is a vertex of $(n N)^{1}$ we let $h_{1}=h$. Suppose then that $h(v)$ is not a vertex. Let $C$ be the closure in $\left|(m M)^{1}\right|$ of the component $Q$ of $\left|(m M)^{1}\right|-T_{M}$ containing $v$; then $C$ is either an arc with endpoints in $T_{M}$ or a simple closed curve which one easily shows must be of the form $Q \cup\{m\}$.

Suppose first it is an arc with endpoints $x$ and $y$. Using the fact that $v$ has order 2 in $\left|(m M)^{1}\right|$ we conclude that one of $x, y$, say $x$, is $m$ and that $Q$ contains no vertex of $m M$ other than $v$.

Let $\sigma$ be the 1-simplex of $m M$ spanned by $m$ and $y$. Applying $h$, we get a pair of $\operatorname{arcs} h(|\sigma|)$ and $h(C)$ in $\left|(n N)^{1}\right|$ whose union is a simple closed curve containing exactly two points of $T_{N}$-namely $h(m)$ and $h(y)$. It follows that there is a vertex $w$ of $n N$ which lies either on $h(|\sigma|-\{m, y\})$ or $h(C-\{m, y\})$. In the first case we choose a selfhomeomorphism $k$ of $\left|(n N)^{1}\right|$ which is the identity off $h(|\sigma| \cup C)$, interchanges $h(|\sigma|)$ and $h(C)$ leaving $h(m)$ and $h(y)$ fixed, and takes $h(v)$ onto $w$; we define $h_{1}=k \circ h$. The second case is similar-except that $k$ is taken as the identity off $h(Q)$.

If $C$ is a simple closed curve, $C=Q \cup\{m\}$, then $Q$ must contain 
exactly two vertices of order 2 , say $v$ and $w$. Since $h(C) \cap T_{N}=\{h(m)\}$ it follows that $h(m)=n$ and $h(Q)$ contains exactly two vertices of order 2, say $v^{\prime}$ and $w^{\prime}$. In this case we choose a self-homeomorphism $k$ of $\left|(n N)^{1}\right|$ which is the identity off $h(Q)$ and takes $h(v)$ to $v^{\prime}$ and $h(w)$ to $w^{\prime}$. The composition $h_{1}=k \circ h$ has the desired properties. This completes the construction of $h_{1}$.

We let $\tilde{h}=h_{r}$; then $\widetilde{h}$ takes each vertex of $(m M)^{1}$ to a veriex of $(n N)^{1}$. In particular $(m M)^{1}$ has at least as many vertices as $(n N)^{1}$. Since a similar construction can be made starting with $h^{-1}$, the number of vertices in each complex is the same. Hence the homeomorphism $\widetilde{h}$ takes the vertices of $(m M)^{1}$ onto those of $(n N)^{1}$. It follows (see, for example, the argument of Theorem 3 of [4]) that $\tilde{h}$ induces an isomorphism of $(m M)^{1}$ onto $(n N)^{1}$.

Applying part (b) of the lemma, we have that $M^{1}$ and $N^{1}$ are isomorphic.

Definition. An $n$-complex $M$ is full provided, for any subcomplex $K$ of $M$ which is isomorphic to $s_{p}^{1}, 2 \leqq p \leqq n, K^{0}$ spans a $p$ simplex of $M$.

THEOREM 2. If $M$ and $N$ are full complexes, then they are isomorphic if $\left|(m M)^{1}\right|$ and $\left|(n N)^{1}\right|$ are homeomorphic.

This follows from Theorem 1 and Theorem 1 of [3] which says that if $M$ and $N$ are full and $M^{1}$ and $N^{1}$ are isomorphic, then $M$ and $N$ are isomorphic.

Definition. Given the cone at $m$ over $M$ and a subcomplex $K$ of $M$ we define the tower-complex over $K$ (relative to $m M$ ) to be $\left((m K)^{\prime}\right)^{1}$ and we denote it by $t_{m}(K)$. Furthermore, we call the underlying polyhedron of $t_{m}(K)$ the tower over $K$ (relative to $m M$ ) and denote it by $t(K)$, i.e.., $t(K)=\left|t_{m}(K)\right|$.

TheOREM 3. If $M$ and $N$ are complexes, then $M$ and $N$ are isomorphic if and only if $t(M)$ and $t(N)$ are homeomorphic.

Proof. Suppose $t(M)$ and $t(N)$ are homeomorphic. We first assume that $M$ and $N$ have no vertices of order 0 . Then the order of each vertex of $(m M)^{\prime}$ in $t_{m}(M)$ and of $(n N)^{\prime}$ in $t_{m}(N)$ is at least three. So we may apply Theorem 5 of [4] to obtain an isomorphism between $m M$ and $n N$. This by part (a) of the Lemma yields the desired isomorphism between $M$ and $N$.

Now consider the case in which $M$ or $N$ has vertices of order 0 . Let $K$ denote the set of vertices of $M$ which are of order 0 and let 
$L$ be the corresponding set for $N$. Let $\widetilde{M}=M-K$ and $\tilde{N}=N-L$. Then

$$
t(M)=t(\tilde{M}) \cup t(K)
$$

and

$$
t(N)=t(\tilde{N}) \cup t(L) .
$$

Let $h$ be a homeomorphism of $t(M)$ onto $t(N)$. Since $t(K)$ is the smallest connected subset of $t(M)$ that contains $K$, the set $h(t(K))$ is the smallest connected subset of $t(N)$ that contains $h(K)$. But $h(K)=L$, because the points of $K$ and $L$ are the only ones with order 1 in $t(M)$ and $t(N)$. Therefore, $h(t(K))=t(L)$, and by taking complements we see that $h(t(\tilde{M}))=t(\tilde{N})$. Therefore, by the preceding argument, there exists an isomorphism $f$ of $\widetilde{M}$ onto $\widetilde{N}$. Since $h$ yields an isomorphism of $K$ and $L, f$ can be extended to an isomorphism of $M$ and $N$.

\section{REFERENCES}

1. R. L. Finney, The insufficiency of barycentric subdivision, Michigan Math. J. 12 (1965), 263-272.

2. P. J. Hilton and S. Wylie, Homology Theory, Cambridge University Press, Cambridge, 1960 .

3. J. Segal, Isomorphic complexes, Bull. Amer. Math. Soc. 71 (1965), 571-572.

4. ___ Isomorphic complexes, II, Bull. Amer. Math. Soc. 72 (1966), 300-302.

Received August 4, 1966. The authors were supported by National Science Foundation grants NSFG-GP3902 and GP5935, respectively.

UNIVERSITY OF WASHINGTON AND

UNIVERSITY OF MICHIGAN 


\section{PACIFIC JOURNAL OF MATHEMATICS}

\section{EDITORS}

\section{H. SAMELSON}

Stanford University

Stanford, California

J. P. JANS

University of Washington

Seattle, Washington 98105

\section{J. DugundJI}

University of Southern California Los Angeles, California 90007

RICHARD ARENS

University of California

Los Angeles, California 90024

\section{ASSOCIATE EDITORS}

E. F. BECKENBACH
B. H. NeUmanN

\section{SUPPORTING INSTITUTIONS}

UNIVERSITY OF BRITISH COLUMBIA CALIFORNIA INSTITUTE OF TECHNOLOGY

UNIVERSITY OF CALIFORNIA

MONTANA STATE UNIVERSITY

UNIVERSITY OF NEVADA

NEW MEXICO STATE UNIVERSITY

OREGON STATE UNIVERSITY

UNIVERSITY OF OREGON

OSAKA UNIVERSITY

UNIVERSITY OF SOUTHERN CALIFORNIA

\author{
STANFORD UNIVERSITY \\ UNIVERSITY OF TOKYO \\ UNIVERSITY OF UTAH \\ WASHINGTON STATE UNIVERSITY \\ UNIVERSITY OF WASHINGTON \\ AMERICAN MATHEMATICAL SOCIETY \\ CHEVRON RESEARCH CORPORATION \\ TRW SYSTEMS \\ NAVAL ORDNANCE TEST STATION
}

Mathematical papers intended for publication in the Pacific Journal of Mathematics should be typewritten (double spaced). The first paragraph or two must be capable of being used separately as a synopsis of the entire paper. It should not contain references to the bibliography. Manuscripts may be sent to any one of the four editors. All other communications to the editors should be addressed to the managing editor, Richard Arens at the University of California, Los Angeles, California 90024.

50 reprints per author of each article are furnished free of charge; additional copies may be obtained at cost in multiples of 50 .

The Pacific Journal of Mathematics is published monthly. Effective with Volume 16 the price per volume ( 3 numbers) is $\$ 8.00$; single issues, $\$ 3.00$. Special price for current issues to individual faculty members of supporting institutions and to individual members of the American Mathematical Society: $\$ 4.00$ per volume; single issues $\$ 1.50$. Back numbers are available.

Subscriptions, orders for back numbers, and changes of address should be sent to Pacific Journal of Mathematics, 103 Highland Boulevard, Berkeley 8, California.

Printed at Kokusai Bunken Insatsusha (International Academic Printing Co., Ltd.), 7-17, Fujimi 2-chome, Chiyoda-ku, Tokyo, Japan.

\section{PUBLISHED BY PACIFIC JOURNAL OF MATHEMATICS, A NON-PROFIT CORPORATION}

The Supporting Institutions listed above contribute to the cost of publication of this Journal, but they are not owners or publishers and have no responsibility for its content or policies. 


\section{Pacific Journal of Mathematics}

\section{Vol. 22, No. $2 \quad$ February, 1967}

Paul Frank Baum, Local isomorphism of compact connected Lie groups ....

Lowell Wayne Beineke, Frank Harary and Michael David Plummer, On the

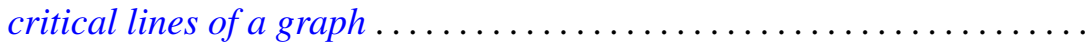

Larry Eugene Bobisud, On the behavior of the solution of the telegraphist's equation for large velocities .......................... 213

Richard Thomas Bumby, Irreducible integers in Galois extensions . . . . . . 221

Chong-Yun Chao, A nonimbedding theorem of nilpotent Lie algebras ..... 231

Peter Crawley, Abelian p-groups determined by their Ulm sequences ...... 235

Bernard Russel Gelbaum, Tensor products of group algebras ........... 241

Newton Seymour Hawley, Weierstrass points of plane domains .......... 251

Paul Daniel Hill, On quasi-isomorphic invariants of primary groups . . . . . 257

Melvyn Klein, Estimates for the transfinite diameter with applications to confomral mapping ................................ 267

Frederick M. Lister, Simplifying intersections of disks in Bing's side approximation theorem ............................. 281

Charles Wisson McArthur, On a theorem of Orlicz and Pettis ........... 297

Harry Wright McLaughlin and Frederic Thomas Metcalf, An inequality for

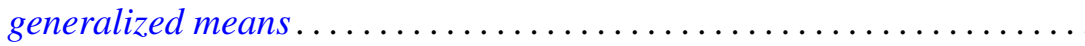

Daniel Russell McMillan, Jr., Some topological properties of piercing

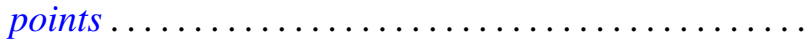

Peter Don Morris and Daniel Eliot Wulbert, Functional representation of topological algebras .

Roger Wolcott Richardson, Jr., On the rigidity of semi-direct products of Lie algebras..................................

Jack Segal and Edward Sandusky Thomas, Jr., Isomorphic

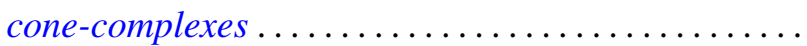

Richard R. Tucker, The $\delta^{2}$-process and related topics.... 